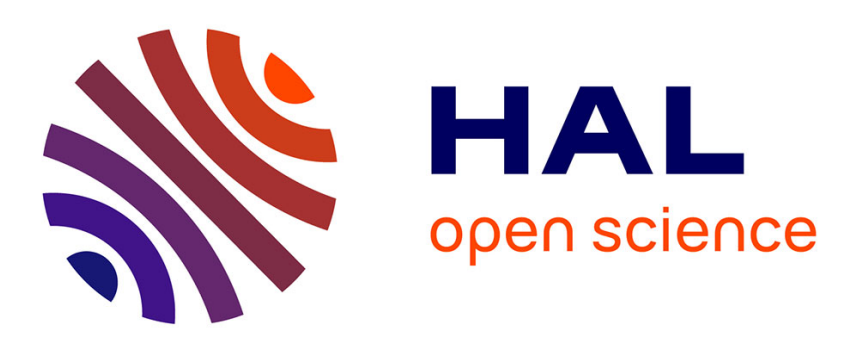

\title{
Avancées dans les tumeurs cérébrales primitives malignes de l'adulte: quels patients transférer en réanimation médicale?
}

Agusti Alentorn, Nicolas Weiss, Jean-Yves Delattre, Ahmed Idbaih

\section{To cite this version:}

Agusti Alentorn, Nicolas Weiss, Jean-Yves Delattre, Ahmed Idbaih. Avancées dans les tumeurs cérébrales primitives malignes de l'adulte: quels patients transférer en réanimation médicale?. Réanimation, 2015, pp.1-9. 10.1007/s13546-015-1073-3 . hal-01187608

\section{HAL Id: hal-01187608 \\ https://hal.sorbonne-universite.fr/hal-01187608}

Submitted on 27 Aug 2015

HAL is a multi-disciplinary open access archive for the deposit and dissemination of scientific research documents, whether they are published or not. The documents may come from teaching and research institutions in France or abroad, or from public or private research centers.
L'archive ouverte pluridisciplinaire HAL, est destinée au dépôt et à la diffusion de documents scientifiques de niveau recherche, publiés ou non, émanant des établissements d'enseignement et de recherche français ou étrangers, des laboratoires publics ou privés. 


\section{Avancées dans les tumeurs cérébrales primitives malignes de l'adulte, Quels patients transférer en réanimation médicale?}

\section{Adult patients with primary malignant brain tumor and admission to intensive care unit}

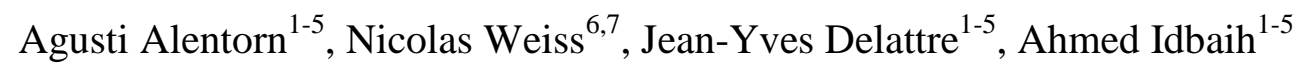

1 AP-HP, Groupe Hospitalier Pitié-Salpêtrière, Service de neurologie 2-Mazarin, Paris, France.

2 Sorbonne Universités, UPMC Univ Paris 06, UM 75, ICM, F-75013 Paris, France

3 Inserm, U 1127, ICM, Paris, F-75013 Paris, France

4 CNRS, UMR 7225, ICM, Paris, F-75013 Paris, France

5 ICM, Paris, 75013 France

6 AP-HP, Unité de Réanimation Neurologique, Fédération de Neurologie 1, Pôle des Maladies du Système Nerveux Groupe Hospitalier Pitié-Salpêtrière 47-83, boulevard de l'Hôpital 75013 PARIS, France

7 Institut de Neurosciences Translationnelles, IHU-A-ICM, PARIS, France

Auteur correspondant: Ahmed Idbaih. Service de Neurologie 2-Mazarin, Groupe Hospitalier Pitié-Salpêtrière. 47-83, Boulevard de l'Hôpital, 75013 Paris, France. Tel: 01-42-16-03-85. Fax: 01-42-16-04-18. Email: ahmed.idbaih@gmail.com or ahmed.idbaih@psl.aphp.fr

Remerciements : Ce travail a bénéficié d'une aide de l'Etat « Investissements d'avenir » ANR-10-IAIHU-06. Institut Universitaire de Cancérologue (IUC).

Titre court : tumeurs cérébrales primitives malignes de l'adulte et réanimation 


\section{Résumé}

Les progrès thérapeutiques réalisés ces dernières années ont permis d'améliorer de manière significative le pronostic et la qualité de vie des patients souffrant de tumeur cérébrale primitive maligne (TCPM) au prix parfois d'une prise en charge transitoire en réanimation médicale. En effet, les patients présentant une TCPM peuvent présenter au cours de leur maladie une défaillance d'organe en rapport avec : (i) une décompensation d'une affection chronique préexistante, (ii) une pathologie aiguë indépendante de leur TCPM, (iii) une complication de la TCPM et/ou, (iv) une complication des traitements de la TCPM. Le bénéfice, immédiat et à long terme, d'un séjour en réanimation pour les patients souffrant spécifiquement de TCPM n'est pas clairement documenté dans la littérature. Une étude dédiée, intégrant notamment les progrès thérapeutiques récents dans la thématique, permettrait de lever le frein historique associé à la prise en charge en réanimation des patients présentant une TCPM. En l'absence de standard de prise en charge médicale, le transfert en réanimation nécessite une discussion collégiale entre le neuro-oncologue, le réanimateur, les proches du patient et la personne de confiance du patient. Cette discussion devra également intégrer les directives anticipées du patient consignées lors de la consultation d'annonce, le pronostic et les perspectives thérapeutiques de la TCPM et de l'affection aigue. Des études cliniques, idéalement prospectives, permettraient de moduler avec un niveau de preuve supérieur ces recommandations.

Mots clés : tumeur cérébrale primitive, neuro-oncologie, réanimation, glioblastome, lymphome cérébral 


\begin{abstract}
Recent therapeutic advances have allowed improving prognosis and quality of life of adult patients suffering from malignant primary brain tumors (MPBT) sometimes after a transient medical support in intensive care unit (ICU). Indeed, patients with MPBT may have, during the course of their neuro-oncological disease, organ(s) failure due to: (i) a destabilization of pre-existing chronic conditions, (ii) an acute pathology unrelated to MPBT (iii) a complication of MPBT and/or, (iv) complications induced by anti-tumor treatments. The short-term and the long term-benefits of an ICU stay for patients with MPBT are not clearly documented in the literature. A dedicated study, including recent advances in the field of MPBT, would participate to remove the historical brake associated with admission of MPBT patients in ICU. Due to the lack of medical and scientific evidences, admission of MPBT patients in ICU requires a multidisciplinary discussion between the neuro-oncologist, the intensivist, the relatives of the patient and the family of the patient. This discussion will also incorporate patient's advanced directives registered during the first visit, the prognosis and the therapeutic perspectives of the MPBT and the acute affection. Further prospective clinical studies will modulate with higher level of evidence these recommendations.
\end{abstract}

Keywords: brain tumor, neuro-oncology and intensive care unit 


\section{Introduction}

Les tumeurs cérébrales primitives (TCP) de l'adulte, dont l'incidence est estimée à environ 10000 nouveaux cas par en France, sont classées selon l'organisation mondiale de la santé (OMS) en cinq grandes catégories en fonction de l'origine embryologique supposée de la cellule initiatrice et de la localisation anatomique: (i) les tumeurs neuroépithéliales, (ii) les tumeurs méningées, (iii) les tumeurs hématopoïétiques, (iv) les tumeurs germinales et, (v) les tumeurs de la région hypothalamo-hypophysaire [1]. Au sein de ces cinq grandes catégories, plus de cent sous-catégories sont individualisées $[1]$.

Sur le plan épidémiologique, les tumeurs neuro-épithéliales sont les TCP les plus fréquentes chez l'adulte. Elles représentent près de 50\% de l'ensemble des TCP. Les tumeurs méningées, les tumeurs de la région sellaire, les tumeurs hématopoïétiques, et les tumeurs germinales sont plus rares et représentent respectivement $\sim 36 \%, \sim 15 \%$, $\sim 3 \%, \sim 1 \%$ de l'ensemble des TCP de l'adulte respectivement [2].

Le diagnostic de certitude de TCP repose sur l'examen neuropathologique d'un fragment de tissu tumoral obtenu lors d'une biopsie cérébrale ou lors d'une résection chirurgicale. Le traitement des patients souffrant de TCP repose sur la chirurgie, la radiothérapie et/ou la chimiothérapie cytotoxique [3].

Le diagnostic de cancer et de tumeurs cérébrales primitives malignes (TCPM) est souvent associé à un pronostic défavorable ce qui constitue le principal frein à une admission en réanimation médicale [4]. Le pronostic des patients souffrant de TCPM, au sein d'un même sous-groupe pathologique, est cependant très hétérogène et très difficile à apprécier en pratique clinique pour un patient donné. Ce pronostic n'est pas 
systématiquement sombre. En effet, y compris dans les TCPM les plus agressives, un nombre non négligeable de patients survivent au-delà de 5 ans, notamment grâce aux avancées thérapeutiques réalisées cette dernière décennie [5,6].

Ces dernières années ont également été marquées par l'incorporation des caractéristiques moléculaires des TCPM dans la prise en charge médicale des patients [7-9]. Ces données moléculaires permettent une meilleure évaluation pronostique, une meilleure planification du traitement et guident les progrès thérapeutiques via des essais cliniques.

De plus, les avancées majeures (i.e. diagnostic et traitements non invasifs) réalisées ces dernières décennies dans les stratégies de réanimation ont permis d'améliorer le pronostic des patients souffrant de cancer [10]. Le taux de mortalité globale des patients oncologiques est passé de $90 \%$ en 1990 , à $50 \%$ en 2010 , [11,12] se rapprochant progressivement de celui de la population générale des patients en réanimation. Cette amélioration du pronostic des patients oncologiques en réanimation est également observée même en cas de défaillance neurologique [13]. Ces résultats sont à nuancer par un probable biais de sélection de la part des oncologues n'orientant pas les patients présentant une défaillance neurologique.

L'hétérogénéité pronostique des TCPM, les progrès thérapeutiques récents et les données moléculaires, devant être intégrées notamment pour guider le transfert en réanimation médicale d'un patient souffrant de TCPM, seront illustrées, ici, via deux grandes catégories de TCPM: (i) les tumeurs gliales et (ii) les lymphomes primitifs du système nerveux central. L'ensemble de ces données nous conduisent à discuter les critères d'admission des patients souffrant de TCPM en réanimation en essayant d'identifier, malgré l'hétérogénéité des TCPM et des situations pouvant aboutir à une 
rea140030_R1

admission en réanimation, les patients qui peuvent bénéficier réellement d'une prise en charge en réanimation. 


\section{Les différents types de tumeurs cérébrales ont un pronostic hétérogène}

\subsection{Les tumeurs gliales}

Les tumeurs gliales diffuses représentent environ 50\% de l'ensemble des TCP. Elles sont classées par l'organisation mondiale de la santé selon le phénotype des cellules tumorales par analogie aux cellules macrogliales normales (i.e. astrocytome, oligodendrogliome et oligoastrocytome) et le grade de malignité de I à IV. Le grade de malignité est un critère semi-quantitatif qui tient compte de la densité cellulaire, de la néoangiogénèse, de la présence de mitose, de la présence de nécrose, et du degré de différentiation cellulaire. La prise en compte récente de données génétiques permet de préciser le pronostic.

Les gliomes de grade I, dominés par les astrocytomes pilocytiques, forment une entité singulière en raison de leur caractère potentiellement curable. Les gliomes de grade II, III et IV sont regroupés sous le terme de gliome diffus et évoluent inexorablement, avec un délai extrêmement variable, vers des tumeurs plus volumineuses ou plus agressives menaçantes pour les pronostics fonctionnels et vitaux des patients. Les gliomes diffus sont donc, en ce sens, des tumeurs malignes.

Les astrocytomes pilocytiques touchent préférentiellement l'enfant et l'adulte jeune. Ils sont caractérisés classiquement à l'IRM par une lésion kystique avec un nodule mural prenant fortement le produit de contraste. Ils sont localisés dans les régions sous tentorielles dans $40 \%$ et en supratentorielles dans $40 \%$. Les autres localisations, plus rares, sont essentiellement la moelle épinière et les nerfs optiques. Une résection chirurgicale complète permet une guérison définitive dans la grande majorité des cas. Néanmoins, dans un certain de cas, l'exérèse complète est impossible. Le traitement repose alors sur la radiothérapie et/ou la chimiothérapie en cas d'évolutivité tumorale. Le taux de survie globale à 5 ans est supérieur à $80 \%$. Les principaux facteurs 
pronostiques cliniques indépendants sont l'âge au diagnostic, l'étendue de la résection chirurgicale, et la réalisation d'une radiothérapie [14]. Sur le plan moléculaire ces tumeurs sont caractérisées par la présence d'anomalies génétiques (e.g. mutation ou réarrangement) activatrices du gène $B R A F$, (B-Raf proto-oncogene, serine/thréonine kinase) localisé sur le chromosome 7, ouvrant des perspectives thérapeutiques intéressantes pour les années à venir avec le développement des inhibiteurs de la voie MAPK dont BRAF est un acteur $[15,16]$.

Les gliomes de grade II ou gliome diffus de bas grade sont des tumeurs malignes lentement évolutives par rapport aux gliomes diffus de grade de malignité supérieur. Elles représentent environ $15 \%$ des tumeurs gliales et touchent principalement l'adulte jeune entre 20 et 40 ans. Ils apparaissent généralement en hyposignal T1, ne prenant pas le contraste sur les séquences injectées et en hypersignal T2 (Figure 1). Leur pronostic est extrêmement hétérogène avec une médiane de survie globale oscillant entre 5 et 15 ans en fonction du sous-type clinico-histo-radio-moléculaire. Le traitement de première ligne des gliomes diffus de bas grade repose sur la chirurgie aussi large que possible. Pour les patients présentant un gliome de bas grade inopérable et présentant des facteurs pronostiques défavorables et/ou une rapide évolutivité, la chimiothérapie cytotoxique et/ou la radiothérapie sont indiquées. Les principaux facteurs pronostiques cliniques au diagnostic des patients souffrant d'un gliome diffus de bas grade sont: (i) l'âge, (ii) l'indice de performance selon le score de performance de Karnofsky ou le score de performance de l'OMS, (iii) le phénotype des cellules tumorales -i.e. astrocytaire, oligodendrocytaire ou mixte-, (iv) la taille tumorale, (v) le franchissement de la ligne médiane, (vi) le statut neurologique selon l'échelle du Medical Research Council, (vii) le statut cognitif idéalement évalué via un bilan neuropsychologique et (viii) la vitesse de croissance tumorale [17-20]. Le statut neurologique et A ces facteurs pronostiques 
clinico-histo-radiologiques, s'ajoutent des facteurs pronostiques génétiques : (i) la codélétion des bras chromosomiques $1 \mathrm{p}$ et $19 \mathrm{q}$, et (ii) les mutations des gènes $I D H 1$ et IDH2 (isocitrate deshydrogénase 1 et 2) codant pour des protéines impliquées dans le métabolisme cellulaire. La combinaison de ces facteurs moléculaires permet d'identifier trois sous-groupes moléculaires de gliomes diffus de bas grade pertinents sur le plan pronostiques: (i) les tumeurs $I D H$ mutées et $1 \mathrm{p} / 19 \mathrm{q}$ co-délétées, (ii) les tumeurs $I D H$ mutées et $1 \mathrm{p} / 19 \mathrm{q}$ non co-délétées et (iii) les tumeurs $I D H$ non mutées et $1 \mathrm{p} / 19 \mathrm{q}$ non codélétées. La médiane de survie est respectivement de 14, 9 et 6 ans [21-23].

Les gliomes de grade III ou gliomes anaplasiques représentent environ $25 \%$ des gliomes. Ils touchent des sujets plus âgés entre 40 et 60 ans. Ces tumeurs sont, ellesaussi, très hétérogènes sur le plan pronostique avec survie globale médiane variant de quelques mois à plusieurs dizaines d'années. Les facteurs pronostiques clinico-historadiologiques sont: (i) l'âge, (ii) l'indice de performance, (iii) la localisation lésionnelle, (iv) le phénotype des cellules tumorales [24-26]. Là aussi la co-délétion des bras chromosomiques $1 \mathrm{p} / 19 \mathrm{q}$ et les mutations des gènes $I D H$ ont une valeur pronostique et prédictive de la réponse au traitement oncologique. Le traitement des gliomes anaplasiques est dorénavant guidé par les données moléculaires. En effet, les tumeurs anaplasiques 1p/19q co-délétées et/ou IDH mutées sont traitées, après la phase chirurgicale, par radiothérapie et chimiothérapie adjuvante ou néoadjuvante par PCV (Procarbazine, Lomustine/CCNU et Vincristine) [9,27]. Pour les tumeurs, ne présentant pas ces altérations, le traitement repose sur la radiothérapie. La place de la chimiothérapie, dans ce dernier sous-groupe de gliome anaplasique, est en cours d'évaluation dans le cadre d'un essai clinique de phase III (NCT00626990). La médiane de survie des patients présentant un gliome anaplasique 1p/19q codélété est de 15 ans. Celle des patients souffrant d'un gliome anaplasique 1p/19q intact et $I D H$ muté est de 6 
ans. Le pronostic est moins favorable dans les gliomes anaplasiques ne présentant aucune de ces deux altérations génétiques avec survie globale médiane entre 12 et 18 mois [28].

Les glioblastomes ou astrocytomes de grade IV représentent environ $25 \%$ de 1'ensemble des TCPM de l'adulte et sont donc le sous-type histologique de gliome diffus le plus fréquent. Environ 1500 à 2000 nouveaux cas sont diagnostiqués chaque année en France [21]. Sur le plan radiologique, ces tumeurs se présentent sous la forme de lésions hétérogènes prenant le contraste en périphérie avec un hyposignal T1 (Figure 1). Les facteurs pronostiques cliniques majeurs sont l'âge et l'indice de performance. L'étendue de la résection chirurgicale et la présence ou non de troubles cognitifs au diagnostic jouent également un rôle pronostique significatif [29]. Le traitement des patients jeunes ( $<70$ ans) en bonne forme (indice de performance $\geq 70 \%$ ) repose sur la radiothérapie associée à une chimiothérapie concomitante puis une chimiothérapie adjuvante par témozolomide. Cette dernière stratégie, validée dans le cadre d'un essai clinique de phase III randomisé contrôlé, a permis une augmentation du taux de survivants à 2 ans de $10 \%$ à $27 \%$ [5]. Ce protocole thérapeutique est communément appelé, protocole Stupp, du nom du premier auteur de la publication rapportant l'essai (Tableau 2, Exemple 1). La méthylation du promoteur de $M G M T$, un gène codant pour un enzyme de réparation des lésions induites à l'ADN par les agents de chimiothérapie alkylant, est un facteur pronostique indépendant mais également un facteur prédictif de la réponse à la chimiothérapie par témozolomide [5,7,30]. Contrairement à la co-délétion $1 \mathrm{p} / 19 \mathrm{q}$ et aux mutations des gènes $I D H$, ce biomarqueur n'est cependant pas encore mature pour une utilisation en pratique [31]. Le pronostic des patients souffrant de glioblastome est globalement sombre avec une survie globale médiane de l'ordre de 15 mois chez les patients présentant des facteurs pronostiques favorables [5]. Néanmoins avec le 
traitement combiné par radiochimiothérapie concomitant et chimiothérapie adjuvante une survie longue est possible. En effet, environ $10 \%$ des patients sont survivants à 5 ans [7].

\subsection{Les lymphomes cérébraux primitifs}

Les lymphomes primitifs du système nerveux central (LPSNC) sont des tumeurs rares touchant le cerveau, les méninges, la moelle épinière, les nerfs crâniens, et l'œil. Environ 300 nouveaux cas sont diagnostiqués chaque année en France chez des patients généralement âgés entre 50 et 60 ans. Il s'agit dans la grande majorité des cas de lymphomes diffus à grandes cellules B. Sur 1'IRM, les LPSNC apparaissent sous la forme de lésion en hyposignal $\mathrm{T} 1$ périventriculaire prenant le contraste de manière intense et « cotonneuse ou en boule de neige ». La survie globale médiane des patients souffrant de LPSNC est aux alentours de 3 ans. Cette médiane cache une très grande hétérogénéité. En effet, la médiane de survie globale des patients âgés de moins de 50 ans est plus proche de 9 ans quant à celle des sujets âgés en mauvais forme clinique est voisine de 1 an. Les facteurs pronostiques cliniques majeurs sont donc l'âge et l'indice de performance. Le nombre de patients longs survivants à 5 ans est important de l'ordre 20 à $40 \%$ selon les études. Le traitement de première ligne repose sur une polychimiothérapie à base de Méthotrexate à haute dose potentiellement source de toxicités rénale, hépatique et hématologique. La place de la chimiothérapie intensive avec autogreffe de cellules souches hématopoïétiques et de la radiothérapie sont discutées au cas par cas et sont actuellement en cours d'évaluation dans des essais cliniques [32-34]. 
rea140030_R1

\section{Principales avancées récentes en termes de traitement et perspectives à court} terme

La radiochimiothérapie concomitante a été une avancée significative dans le traitement des patients souffrant de glioblastome en triplant le nombre de survivants à 2 ans [6]. De même, l'adjonction précoce, par rapport à la radiothérapie, d'une chimiothérapie par procarbarzine, lomustine et vincristine a permis de doubler la survie globale des patients présentant une tumeur oligodendrogliale anaplasique $1 \mathrm{p} / 19 \mathrm{q}$ codélétée [8,9]. Ce dernier résultat souligne l'incorporation désormais des données moléculaires dans la prise en charge médicale des patients souffrant de tumeurs gliales pour mieux : (i) préciser le diagnostic, (ii) préciser le pronostic et (iii) guider les traitements.

De nombreux traitements anti-tumoraux innovants sont actuellement développés ou en cours de développement. Les plus avancés en neuro-oncologie sont les antiangiogéniques et notamment le bevacizumab, un anticorps monoclonal humanisé antiVEGF-A, évalué chez les patients présentant un glioblastome nouvellement diagnostiqué ou en première récidive. La combinaison lomustine et bevacizumab chez les patients présentant un glioblastome en première récidive semble prometteuse dans le cadre d'un essai clinique de phase II contrôlé randomisé avec un taux de survivants à 9 mois passant de $43 \%$ (dans le bras lomustine seule) à $63 \%$ (dans le bras lomustine plus bevacizumab) [35]. La place du bevacizumab dans le traitement des patients présentant un glioblastome nouvellement diagnostiqué n'est pas clairement établie. Deux essais cliniques de phase III randomisés contrôlés en double aveugle ont montré un allongement de la survie sans progression sans bénéfice en termes de survie globale chez les patients traités par le traitement standard plus bevacizumab versus le traitement standard plus placebo [36,37]. L'impact sur les fonctions cognitives et la qualité de vie, 
de l'adjonction de bevacizumab au traitement standard, chez les patients souffrant d'un glioblastome nouvellement diagnostiqué n'est pas clairement établi. L'European Medicine Agency a récemment rejeté la demande d'autorisation de mise sur le marché du bevacizumab chez les patients présentant un glioblastome nouvellement diagnostiqué.

\section{Circonstances de transfert en réanimation, quels patients transférer ?}

Les patients souffrant de cancer représentent environ $15 \%$ des patients hospitalisés en réanimation et les patients porteurs d'une TCP représentent environ $10 \%$ des patients porteurs d'une tumeur solide admis en réanimation [38,39].

L'incidence des TCPM est globalement stable. En revanche, la survie globale s'allonge au fil des progrès thérapeutiques augmentant ainsi la prévalence de la maladie. Sur une période de temps donnée, le nombre de patients souffrant de TCPM nécessitant une

prise en charge en réanimation est donc croissant. Ainsi, dans notre structure, le nombre de patients admis en réanimation neurologique a quasi-doublé en 20 ans (données non publiées, Tableau 1).

\subsection{Circonstances pouvant justifier de la réanimation}

En dehors de décompensation des co-morbidités (e.g. diabète, bronchopneumopathie chronique obstructive) ou d'apparition d'affections non liées à la maladie neurologique (i.e. infarctus du myocarde, sepsis), les affections aigues pouvant nécessité une hospitalisation en réanimation peuvent être liées à la maladie tumorale elle-même ou aux traitements anti-tumoraux. 


\subsubsection{Aggravations liées à la maladie tumorale}

En effet, un patient présentant une TCPM peut s'aggraver avec l'apparition d'une défaillance neurologique lors de crises comitiales ou d'un état de mal comitial [40-44], d'une progression tumorale plus ou moins rapide intraparenchymateuse ou méningée, d'une hémorragie cérébro-méningée et d'hydrocéphalie aigue responsable d'une hypertension intracrânienne. Dans notre expérience, l'hospitalisation en réanimation pour un état de mal comitial et/ou des crises comitiales représente près de $40 \%$ des admissions en réanimation chez les patients admis pour TCPM (données non publiées). La prise en charge réanimatoire de ces états de mal comitiaux tumoraux n'a rien de spécifique et sont rarement résistants aux traitements conventionnels. En cas d'aggravation tumorale, l'adjonction de corticostéroïdes pour diminuer la part d'œdème cérébrale peut être intéressante. Certains patients atteints de tumeurs gliales de bas grade peuvent présenter des états de mal partiels de plusieurs jours, parfois difficile à contrôler. Il relève d'une prise en charge médicamenteuse spécifique et spécialisée. Le recours à des anesthésiques généraux est rarement nécessaire et en cas de forme résistante au traitement, le traitement de la tumeur peut aider à amender les symptômes comitiaux.

\subsubsection{Aggravations liées au traitement}

La chirurgie diagnostique et/ou thérapeutique couplée à la prise en charge anesthésique peut se compliquer d'une défaillance d'organe nécessitant une prise en charge en réanimation qui n’a rien de spécifique. 
Enfin, les traitements cytotoxiques et les traitements symptomatiques ou préventifs (i.e. corticoïdes, anti-épileptiques, anti-coagulants) peuvent être responsables : (i) d'infections opportunistes (e.g. pneumocystose), (ii) d'infections à germes banals compliquées ou non d'un choc septique, (iii) d'une défaillance hépatique chez les patients avec ou sans une hépatopathie sous-jacente (e.g. témozolomide), (iv) d'une insuffisance rénale aigue (e.g. méthotrexate) [40], (v) d'une insuffisance cardiaque (e.g. anthracyclines), (vi) d'une hémorragie (e.g. bevacizumab) et/ou (vii) de troubles métaboliques. La survenue d'une pneumopathie infectieuse aggravée par d'éventuels troubles de la déglutition et l'immunodépression reste cependant au premier rang. Par ailleurs, ces chimiothérapies sont, pour la plupart, également responsables d'une hématotoxicité (i.e. thrombopénie, leucopénie, anémie) et de nausées/vomissements nécessitant toujours une surveillance clinico-biologique régulière et parfois des traitements préventifs.

\subsection{Quel patient admettre en réanimation?}

A notre connaissance aucune étude clinique n'a permis d'établir des facteurs pronostiques robustes permettant d'identifier spécifiquement les patients souffrant de TCPM pour lesquels un séjour en réanimation a été ou serait bénéfique. Le Tableau 2 montre les caractéristiques et le devenir des patients atteints de TCP admis en réanimation neurologique entre 1995 et 2014 dans notre institution (données non publiées).

En revanche, des facteurs pronostiques ont été explorés dans d'autres tumeurs solides et dans les hémopathies malignes. Ces études, combinées aux études menées en cancérologie, ont permis de dégager plusieurs messages importants guidant l'admission en réanimation d'un patient souffrant de cancer [10,11]. Ces recommandations peuvent 
être raisonnablement, en l'absence d'études spécifiques, étendues aux patients souffrant de TCPM.

De manière générale, l'admission en réanimation médicale des patients souffrant de cancer est conditionnée par l'intégration des perspectives thérapeutiques et pronostiques, en termes de survie et de qualité de vie: (i) de l'affection aigue et des défaillances d'organes associées et, (ii) de la maladie tumorale. Le souhait du patient et de son entourage, notamment de sa personne de confiance, sont également des éléments clés de la décision.

Les perspectives thérapeutiques et pronostiques des patients souffrant de TCPM sont relativement bien établies au diagnostic et en phase terminale de la maladie. Le pronostic au début de la maladie a été le plus souvent établi dans le cadre d'essais cliniques. A la phase terminale, le pronostic est estimé empiriquement à quelques semaines. En revanche, entre ces deux bornes, le pronostic précis est moins bien connu. De plus, les données pronostiques récentes intégrant les données génétiques peuvent être difficiles à intégrer par le réanimateur. Ainsi, une interaction avec l'oncologue ou le neuro-oncologue et le neurochirurgien est le plus souvent utile.

Le pronostic du patient admis en réanimation est relativement difficile à établir, dans les premiers jours post-admission, en dehors de certaines situations particulières : (i) les patients grabataires, (ii) les patients âgés de plus de 70 ans en mauvaise forme [45-48], et (iii) les patients présentant un score de gravité élevé $[48,49]$. Mais ces critères restent imparfaits et nécessitent d'être raffinés [50].

\subsubsection{Situations simples et rares}

Pour les patients présentant une TCPM nouvellement diagnostiquée et une affection aigue nécessitant une admission en réanimation, le transfert en réanimation semble 
indiscutable. En effet, le pronostic de patients présentant un cancer est comparable aux autres patients présentant une comorbidité grave [46,51] et les patients présentant une TCPM ne devraient pas faire exception au vue des données pronostiques détaillées plus haut.

Pour les patients présentant une TCPM évolutive pour laquelle toutes les ressources thérapeutiques oncologiques ont été épuisées, l'admission en réanimation apparait déraisonnable et ceci d'autant plus si l'autonomie et la qualité de vie du patient sont altérées. Dans ce cas de figure, le pronostic est très sombre avec une espérance de vie estimée à quelques semaines.

\subsubsection{Situations complexes et fréquentes}

Dans la grande majorité, les patients souffrant de TCPM et nécessitant un transfert en réanimation sont dans la zone grise entre ces deux situations extrêmes. La décision de prise en charge en réanimation nécessite donc, au cas par cas, une discussion multidisciplinaire entre le réanimateur d'une part et le neuro-oncologue ou le neurochirurgien d'autre part. Cette discussion médicale collégiale doit prendre en compte l'avis du patient s'il peut le verbaliser ou figurant dans ses directives anticipées et celui de sa famille et de sa personne de confiance.

Le pronostic de l'affection aigue nécessitant la prise en charge en réanimation peut être approché par les scores pronostiques de réanimation avec notamment le nombre de défaillances d'organes. Il nous apparaît cependant que l'admission en réanimation pour le traitement de crises convulsives ou d'un état de mal comitial semble être une indication licite. L'admission en réanimation pour une pneumopathie infectieuse nous semble d'autant plus licite en début de maladie ou dans la zone d'incertitude et en cas de survenue en absence de troubles de la déglutition du à la progression tumorale. La 
gravité et le pronostic de la pneumopathie sera fonction de critères de gravité habituelles.

En cas de progression tumorale, les décisions d'admission en réanimation sont plus difficiles. Le pronostic de l'affection tumorale dépend de plusieurs paramètres : (i) le type histo-moléculaire, (ii) l'indice de performance du patient, (iii) l'âge du patient, et (iv) la phase/le stade de la maladie neuro-oncologique. L'intégration de tous ces paramètres pronostiques, en l'absence d'études dédiées, est extrêmement difficile en pratique (Tableau 2). De manière très pragmatique, le concept d'Intensive Care Unit trial (ICU Trial) de quelques jours (e.g. 3 à 5 jours) apparait tout à fait adapté à ces situations complexes et fréquentes (Figure 2) [52]. En effet, le pronostic à l'admission est difficile à établir. En revanche, il apparait plus évident au bout de quelques jours de soins intensifs maximalistes incluant une chimiothérapie si elle apparait justifiée [50]. Alors que dans certaines études [50], tous les patients ayant nécessité une ventilation mécanique ou des drogues vasopressives à 3 jours de l'admission décèdent, il faut noter que les patients ayant une TCPM nécessitent souvent le recours à la ventilation mécanique afin d'assurer la protection des voies aériennes supérieures. L'extrapolation de ces données aux patients atteints de TCPM doit ainsi être réalisée avec prudence. Ces données permettent de moduler la limitation des thérapeutiques actives vers une prise en charge palliative exclusive ou la poursuite des mesures de réanimation quelques jours après l'admission [11].

Dans ces situations, l'admission en réanimation doit se faire au plus vite pour limiter l'accumulation des défaillances d'organes et privilégier les méthodes diagnostiques et thérapeutiques non invasives.

\subsubsection{Situations particulières}


Les décisions de non transfert en réanimation et/ou de limitations des thérapeutiques actives doivent être prises de manière multidisciplinaire en concertation avec les proches et la personne de confiance du patient qui veille aux volontés exprimées par le patient avant son aggravation. Dans la majorité, il y a un consensus. En revanche dans certains cas, les proches, malgré l'absence de perspectives thérapeutiques, souhaitent une réanimation importante. Dans ces cas une admission en réanimation peut parfois permettre à l'équipe médicale de mettre en place les stratégies nécessaires (information, prise en charge psychologique, aide par l'unité de soins palliatifs) afin que la famille puisse adhérer au caractère irréversible et évolutif de l'aggravation.

\section{Conclusion}

Des études portant sur les patients souffrant de TCPM admis ou non en réanimation manquent cruellement dans la littérature limitant le niveau de preuve des recommandations fournies. La décision de transfert est multidisciplinaire impliquant le réanimateur et le neuro-oncologue qui évaluent le bénéfice potentiel d'un transfert en réanimation en fonction de la maladie tumorale et de l'affection aigue. Avec l'adhésion de la personne de confiance du patient, il apparait déraisonnable de transférer en réanimation un patient dont la TCPM est évolutive et pour laquelle toutes les ressources thérapeutiques ont été épuisées et dont le pronostic réanimatoire est sombre (i.e. sujet âgé, sujet grabataire et sujet en défaillance multiviscérale). En l'absence d'adhésion de la personne de confiance du patient, une admission en réanimation donne parfois le temps nécessaire pour obtenir un consensus sur la limitation des thérapeutiques actives. Certaines situations particulières, et notamment, la survenue de crises convulsives ou d'un état de mal convulsif semblent bénéficier particulièrement d'une admission en 
réanimation chez les malades atteints de TCPM. Dans les autres situations, l'ICU trial avec une réanimation maximaliste, pendant quelques jours, permet de mieux appréhender le pronostic réanimatoire du patient et de prendre une décision de poursuite de la réanimation maximaliste ou de limitation des thérapeutiques actives pour une prise en charge palliative. 


\section{Références}

1. Louis DN, Ohgaki H, Wiestler OD, et al. (2007) The 2007 WHO classification of tumours of the central nervous system. Acta Neuropathol (Berl) 114:97-109.

2. Ostrom QT, Gittleman H, Farah P, et al. (2013) CBTRUS statistical report: Primary brain and central nervous system tumors diagnosed in the United States in 2006-2010. Neuro Oncol Suppl 2:ii1-56.

3. Ricard D, Idbaih A, Ducray F, et al. (2012) Primary brain tumours in adults. Lancet 379:1984-96.

4. [No authors listed] (1999) Guidelines for intensive care unit admission, discharge, and triage. Task Force of the American College of Critical Care Medicine, Society of Critical Care Medicine. Crit Care Med 27:633-8.

5. Stupp R, Mason WP, van den Bent MJ, et al. (2005) Radiotherapy plus concomitant and adjuvant temozolomide for glioblastoma. N Engl J Med 352:98796.

6. Stupp R, Hegi ME, Mason WP, et al. (2009) Effects of radiotherapy with concomitant and adjuvant temozolomide versus radiotherapy alone on survival in glioblastoma in a randomised phase III study: 5-year analysis of the EORTCNCIC trial. Lancet Oncol. 2009 10:459-66.

7. Hegi ME, Diserens AC, Gorlia T, et al. (2005) MGMT gene silencing and benefit from temozolomide in glioblastoma. N Engl J Med 352:997-1003.

8. van den Bent MJ, Erdem-Eraslan L, Idbaih A, et al. (2013) MGMT-STP27 methylation status as predictive marker for response to PCV in anaplastic Oligodendrogliomas and Oligoastrocytomas. A report from EORTC study 26951. Clin Cancer Res 19:5513-22. 
9. Cairncross G, Wang M, Shaw E, et al. (2013) Phase III trial of chemoradiotherapy for anaplastic oligodendroglioma: long-term results of RTOG 9402. J Clin Oncol. $31: 337-43$.

10. Kostakou E, Rovina N, Kyriakopoulou M, et al. (2014) Critically ill cancer patient in intensive care unit: Issues that arise. J Crit Care S0883-9441:00145-2.

11. Azoulay E, Soares M, Darmon M, et al. (2011) Intensive care of the cancer patient: recent achievements and remaining challenges. Ann Intensive Care 1:5.

12. Peigne V, Rusinová K, Karlin L, et al. (2009) Continued survival gains in recent years among critically ill myeloma patients. Intensive Care Med 35:512-8.

13. Legriel S, Marijon H, Darmon M, et al. (2010) Central neurological complications in critically ill patients with malignancies. Intensive Care Med 36:232-40.

14. Johnson DR, Brown PD, Galanis E, Hammack JE (2012) Pilocytic astrocytoma survival in adults: analysis of the Surveillance, Epidemiology, and End Results Program of the National Cancer Institute. J Neurooncol 108:187-93.

15. Bautista F, Paci A, Minard-Colin V, et al. (2014) Vemurafenib in pediatric patients with BRAFV600E mutated high-grade gliomas. Pediatr Blood Cancer 61:1101-3.

16. Skrypek M, Foreman N, Guillaume D, Moertel C (2014) Pilomyxoid astrocytoma treated successfully with vemurafenib. Pediatr Blood Cancer [Epub ahead of print]

17. Pignatti F, van den Bent M, Curran D, et al. (2002) Prognostic factors for survival in adult patients with cerebral low-grade glioma. J Clin Oncol 20:2076-84.

18. Soffietti R, Baumert BG, Bello L, et al. (2010) Guidelines on management of lowgrade gliomas: report of an EFNS-EANO Task Force. Eur J Neurol 17:1124-33.

19. Daniels TB, Brown PD, Felten SJ, et al. (2011) Validation of EORTC prognostic factors for adults with low-grade glioma: a report using intergroup 86-72-51. Int J Radiat Oncol Biol Phys. 81:218-24. 
20. Mandonnet E, Delattre JY, Tanguy ML, et al. (2003) Continuous growth of mean tumor diameter in a subset of grade II gliomas. Ann Neurol. 2003 53:524-8.

21. Rigau V, Zouaoui S, Mathieu-Daudé H, et al. (2011) French brain tumor database: 5-year histological results on 25756 cases. Brain Pathol 21:633-44.

22. Houillier C, Wang X, Kaloshi G, et al. (2010) IDH1 or IDH2 mutations predict longer survival and response to temozolomide in low-grade gliomas. Neurology 75:1560-6.

23. Everhard S, Tost J, El Abdalaoui H, et al. (2009) Identification of regions correlating MGMT promoter methylation and gene expression in glioblastomas. Neuro Oncol 11:348-56.

24. Gorlia T, Delattre JY, Brandes AA, et al. (2013) New clinical, pathological and molecular prognostic models and calculators in patients with locally diagnosed anaplastic oligodendroglioma or oligoastrocytoma. A prognostic factor analysis of European Organisation for Research and Treatment of Cancer Brain Tumour Group Study 26951. Eur J Cancer 49:3477-85.

25. Panageas KS, Reiner AS, Iwamoto FM, et al. (2014) Recursive partitioning analysis of prognostic variables in newly diagnosed anaplastic oligodendroglial tumors. Neuro Oncol [Epub ahead of print]

26. Stupp R, Reni M, Gatta G, et al. (2007) Anaplastic astrocytoma in adults. Crit Rev Oncol Hematol 63:72-80.

27. van den Bent MJ, Brandes AA, Taphoorn MJ, et al. (2013) Adjuvant procarbazine, lomustine, and vincristine chemotherapy in newly diagnosed anaplastic oligodendroglioma: long-term follow-up of EORTC brain tumor group study 26951. J Clin Oncol 31:344-50. 
28. Cairncross JG, Wang M, Jenkins RB, et al. (2014) Benefit from procarbazine, lomustine, and vincristine in oligodendroglial tumors is associated with mutation of IDH. J Clin Oncol. 32:783-90.

29. Li J, Wang M, Won M, et al. (2011) Validation and simplification of the Radiation Therapy Oncology Group recursive partitioning analysis classification for glioblastoma. Int J Radiat Oncol Biol Phys. 81:623-30.

30. Esteller M, Garcia-Foncillas J, Andion E, et al. (2000) Inactivation of the DNArepair gene MGMT and the clinical response of gliomas to alkylating agents. $\mathrm{N}$ Engl J Med 343:1350-4.

31. Tabouret E, Chinot O, Sanson M, et al. (2014) Predictive biomarkers investigated in glioblastoma. Expert Rev Mol Diagn 14:883-93.

32. Korfel A, Schlegel U. (2013) Diagnosis and treatment of primary CNS lymphoma. Nat Rev Neurol 9:317-27.

33. Sierra del Rio M, Rousseau A, Soussain C, et al. (2009) CNS lymphoma in immunocompetent patients. The Oncologist 14:526-39.

34. Abrey LE, Ben-Porat L, Panageas KS, et al. (2006) Primary central nervous system lymphoma: the Memorial Sloan-Kettering Cancer Center prognostic model. J Clin Oncol 24:5711-5.

35. Taal W, Oosterkamp HM, Walenkamp AM, et al. (2014) Single-agent bevacizumab or lomustine versus a combination of bevacizumab plus lomustine in patients with recurrent glioblastoma (BELOB trial): a randomised controlled phase 2 trial. Lancet Oncol 15:943-53.

36. Gilbert MR, Dignam JJ, Armstrong TS, et al. (2014) A randomized trial of bevacizumab for newly diagnosed glioblastoma. N Engl J Med 370:699-708 
37. Chinot OL, Wick W, Mason W, et al. (2014) Bevacizumab plus radiotherapytemozolomide for newly diagnosed glioblastoma. N Engl J Med 370:709-22.

38. Taccone FS, Artigas AA, Sprung CL, et al. (2009) Characteristics and outcomes of cancer patients in European ICUs. Crit Care 13:R15.

39. Soares M, Salluh JI, Torres VB, et al. (2005) Short- and long-term outcomes of critically ill patients with cancer and prolonged ICU length of stay. Chest 134:5206.

40. Cavaliere R, Farace E, Schiff D. (2006) Clinical implications of status epilepticus in patients with neoplasms. Arch Neurol 63:1746-9.

41. Ferlisi M, Shorvon S. (2012) The outcome of therapies in refractory and superrefractory convulsive status epilepticus and recommendations for therapy. Brain J Neurol 135:2314-28.

42. Kennedy J, Schuele SU. (2013) Long-term monitoring of brain tumors: when is it necessary? Epilepsia 54 Suppl 9:50-5.

43. Marcuse LV, Lancman G, Demopoulos A, Fields M. (2014) Nonconvulsive status epilepticus in patients with brain tumors. Seizure 23:542-7.

44. Schmiegelow K. (2009) Advances in individual prediction of methotrexate toxicity: a review. Br J Haematol 146:489-503.

45. Roch A, Wiramus S, Pauly V, et al. (2011) Long-term outcome in medical patients aged 80 or over following admission to an intensive care unit. Crit Care 15:R36.

46. Darmon M, Azoulay E. (2009) Critical care management of cancer patients: cause for optimism and need for objectivity. Curr Opin Oncol 21:318-26.

47. Boussat S, El'rini T, Dubiez A, et al. (2000) Predictive factors of death in primary lung cancer patients on admission to the intensive care unit. Intensive Care Med 26:1811-6. 
48. Kopterides P, Liberopoulos P, Ilias I, et al. (2011) General prognostic scores in outcome prediction for cancer patients admitted to the intensive care unit. Am $\mathbf{J}$ Crit Care 20:56-66.

49. Aygencel G, Turkoglu M, Turkoz Sucak G, Benekli M. (2014) Prognostic factors in critically ill cancer patients admitted to the intensive care unit. J Crit Care 29:618-26.

50. Darmon M, Thiery G, Ciroldi M, et al. (2005) Intensive care in patients with newly diagnosed malignancies and a need for cancer chemotherapy. Crit Care Med 33:2488-93.

51. Thiéry G, Azoulay E, Darmon M, et al. (2005) Outcome of cancer patients considered for intensive care unit admission: a hospital-wide prospective study. J Clin Oncol 23:4406-13.

52. Lecuyer L, Chevret S, Thiery G, et al. (2007) The ICU trial: a new admission policy for cancer patients requiring mechanical ventilation. Crit Care Med 2007 $35: 808-14$. 
rea140030_R1

\section{Légendes}

\section{Figure 1. Aspect radiologique (IRM) de tumeurs cérébrales primitives de l'adulte}

Panel A. Gliome de bas grade (gliome de grade II) en hyposignal sur la séquence T1SE (A1), ne prenant pas le contraste (A2) et en hypersignal sur la séquence T2 FLAIR (A3) Panel B. Gliome de haut grade (glioblastome, grade IV) en hyposignal sur la séquence T1SE (B1), prenant le contraste de manière hétérogène principalement en périphérie (B2) et en hypersignal sur la séquence T2 FLAIR (B3)

Panel C. Lymphome primitif système nerveux central en hyposignal sur la séquence T1SE (C1), prenant le contraste de manière homogène floconneuse en «boule de neige » périventriculaire (C2) et en hypersignal sur la séquence T2 FLAIR (C3)

Figure 2. Proposition d'un algorithme guidant l'admission en réanimation de patients souffrant de tumeur cérébrale primitive maligne et présentant une défaillance d'organe 
rea140030_R1

Figure 1

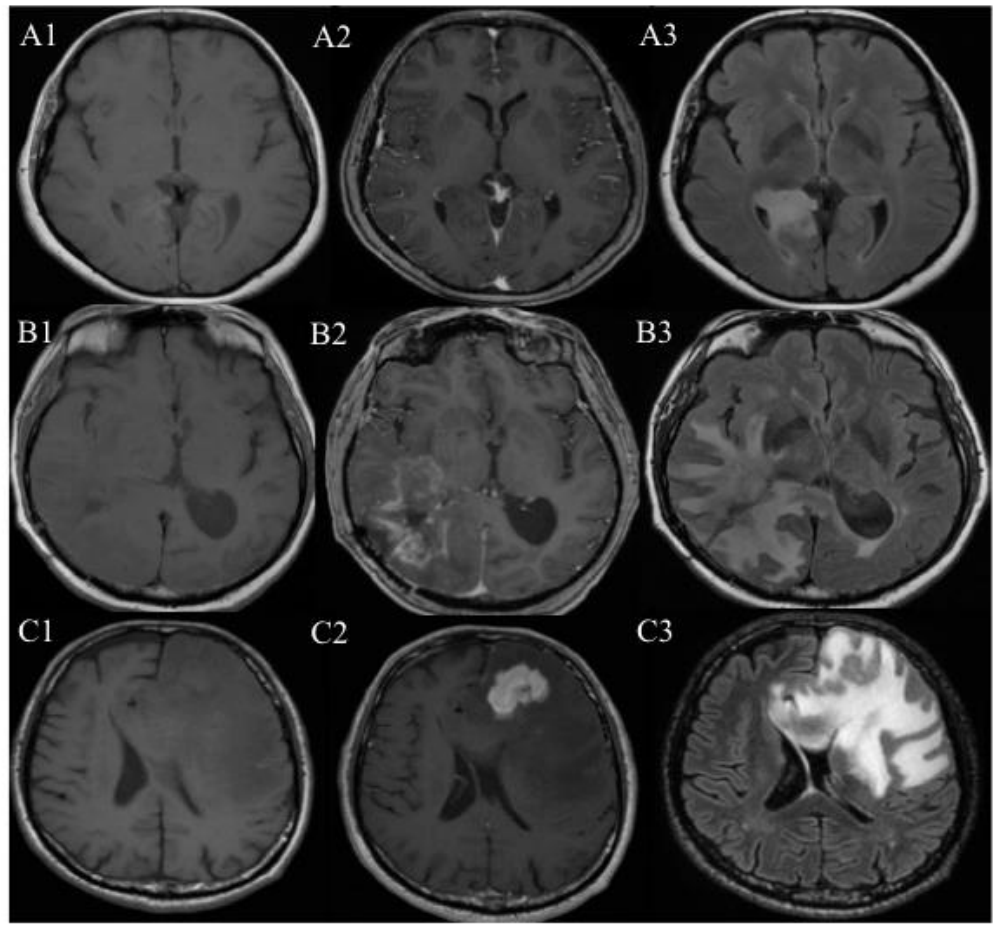

Figure 2

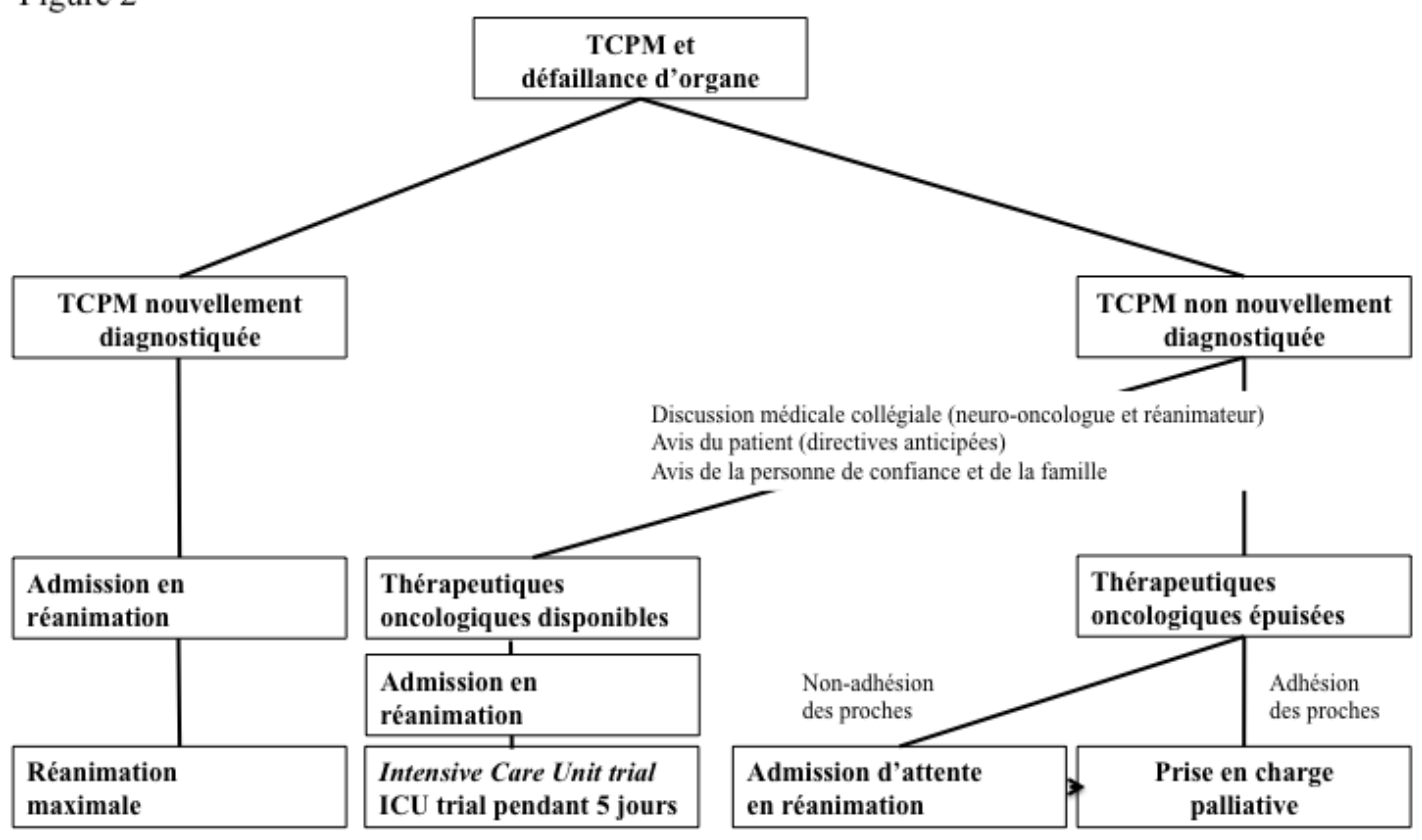


Tableau 1 : patients hospitalisés en réanimation neurologique pour TCP

\begin{tabular}{|l|c|c|}
\hline & Réanimation (1995-2014) & USC (2011-2014) \\
& $\mathbf{n = 1 0 3}$ & $57 \pm 15$ \\
\hline Age (années) & $52 \pm 15$ & $20 \pm 11$ \\
\hline IGS-2 & $37 \pm 21$ & $12 \pm 4$ \\
\hline Score de Glasgow & $9 \pm 4$ & $16(89 \%)$ \\
\hline Mode d'entrée & & $2(11 \%)$ \\
\hline Transfert interne & $64(62 \%)$ & $4 \pm 4$ \\
\hline Entrée directe & $29(28 \%)$ & \\
\hline $\begin{array}{l}\text { Durée moyenne de séjour } \\
\text { (jours) }\end{array}$ & $10 \pm 12$ & $1(6 \%)$ \\
\hline Mode de sortie & & \\
\hline Décès en réanimation ou & & \\
& & \\
\hline
\end{tabular}




\section{Tableau 2 : Deux exemples concrets}

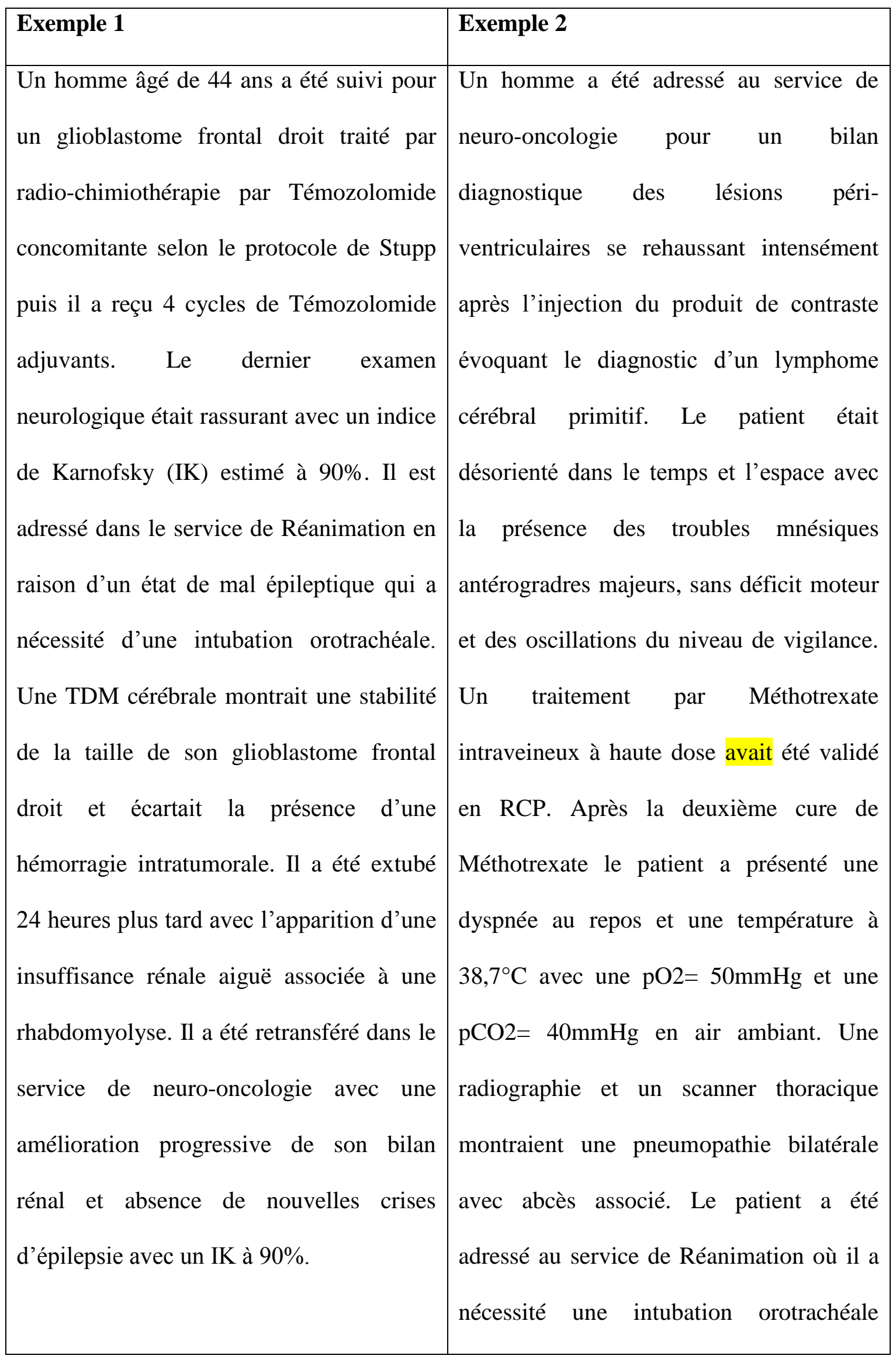




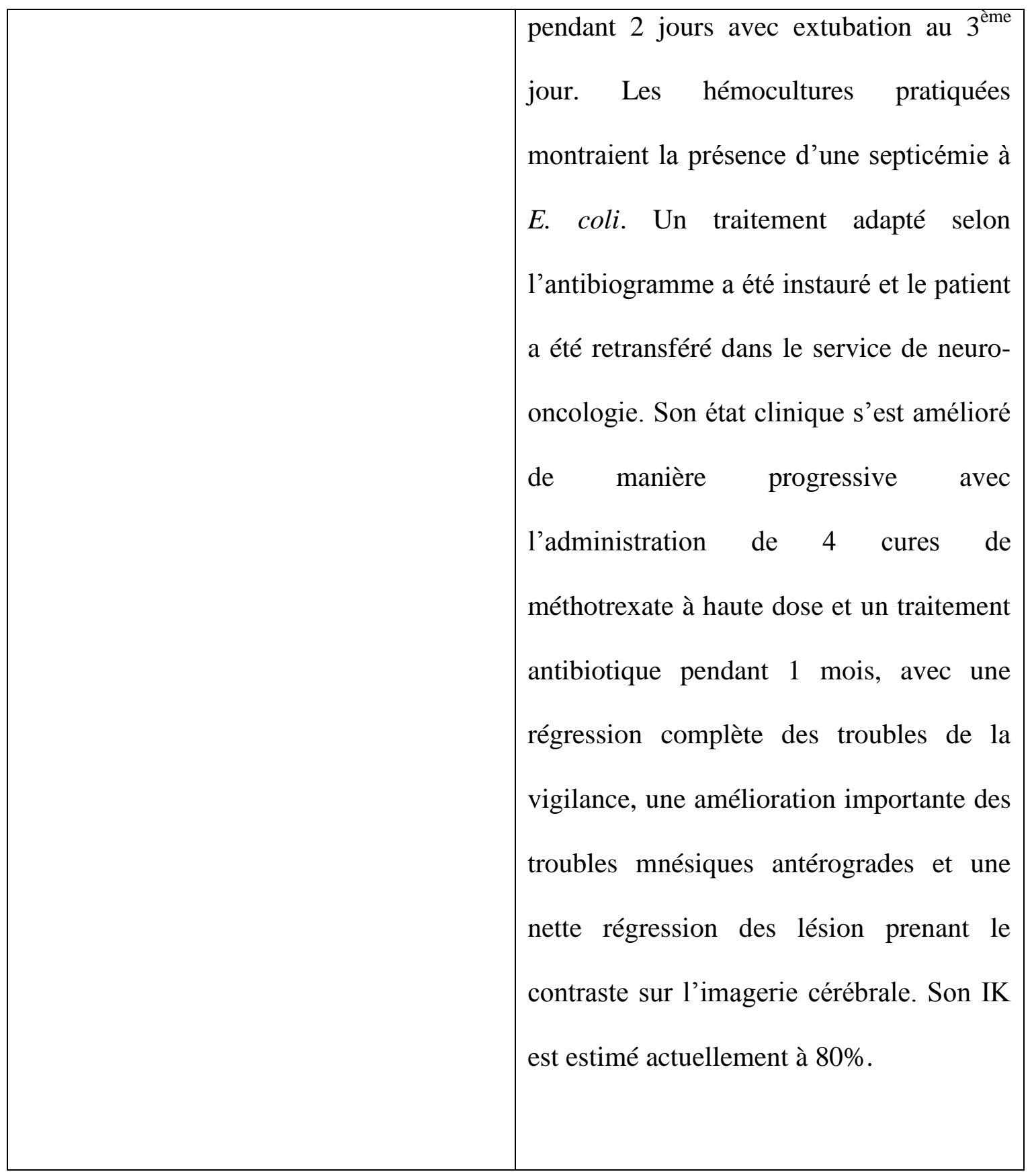

